PROCEEDINGS OF THE

AMERICAN MATHEMATICAL SOCIETY

Volume 126, Number 2, February 1998, Pages 411-420

S 0002-9939(98)04206-3

\title{
COMPOSITION OPERATORS THAT IMPROVE INTEGRABILITY ON WEIGHTED BERGMAN SPACES
}

\author{
WAYNE SMITH AND LIMING YANG \\ (Communicated by Theodore W. Gamelin)
}

\begin{abstract}
Composition operators between weighted Bergman spaces with a smaller exponent in the target space are studied. An integrability condition on a generalized Nevanlinna counting function of the inducing map is shown to characterize both compactness and boundedness of such an operator. Composition operators mapping into the Hardy spaces are included by making particular choices for the weights.
\end{abstract}

\section{INTRODUCTION}

Let $\mathbb{D}$ be the open unit disk in the complex plane and let $\varphi: \mathbb{D} \rightarrow \mathbb{D}$ be an analytic self-map of $\mathbb{D}$. Then $\varphi$ induces a linear operator $C_{\varphi}$ on the space of analytic functions on $\mathbb{D}$, defined by $C_{\varphi}(f)=f \circ \varphi$. The restrictions of such a composition operator to various Banach spaces of analytic functions on $\mathbb{D}$ have received much attention. In particular, it is a well known consequence of Littlewood's Subordination Principle that every composition operator maps every Hardy and Bergman space into itself (see for example [MS] or [Sh]). Here we continue the line of investigation from Hunziker and Jarchow [HJ], Riedl [Ri] and the first author [Sm], and study composition operators that in some sense improve integrability.

We denote Lebesgue measure on $\mathbb{D}$ by $d A$, normalized so that $A(\mathbb{D})=1$. For $\gamma>-1$, define the measure $d A_{\gamma}$ on $\mathbb{D}$ by

$$
d A_{\gamma}(w)=[\log (1 /|w|)]^{\gamma} d A(w) .
$$

For $0<p<\infty$ and $\gamma>-1$ the weighted Bergman space $A_{\gamma}^{p}$ consists of those functions $f$ analytic on $\mathbb{D}$ and satisfying

$$
\|f\|_{A_{\gamma}^{p}}^{p}=\int_{\mathbb{D}}|f(w)|^{p} d A_{\gamma}(w)<\infty .
$$

The same space of functions and an equivalent norm results if the measure $d A_{\gamma}$ is replaced by $(1-|w|)^{\gamma} d A(w)$, since $(1-|w|)^{\gamma}$ and $[\log (1 /|w|)]^{\gamma}$ are comparable for $1 / 2 \leq|w|<1$, and the singularity of $d A_{\gamma}$ at the origin is integrable. The

Received by the editors July 24, 1996.

1991 Mathematics Subject Classification. Primary 47B38; Secondary 30D55, 46E15.

Key words and phrases. Bergman spaces, Hardy spaces, composition operators, Nevanlinna counting function.

The second author was partially supported by National Science Foundation grant DMS9531917 and a seed-money grant from the University of Hawaii.

(C)1998 American Mathematical Society 
unweighted Bergman space $A_{0}^{p}$ will also be denoted simply by $A^{p}$. For $p>0$, the Hardy space $H^{p}$ consists of those functions analytic on $\mathbb{D}$ and satisfying

$$
\|f\|_{H^{p}}^{p}=\lim _{r \rightarrow 1-} \frac{1}{2 \pi} \int_{0}^{2 \pi}\left|f\left(r e^{i \theta}\right)\right|^{p} d \theta<\infty .
$$

J. H. Shapiro in [Sh] used the classical Nevanlinna counting function $N_{\varphi, 1}$ (defined below) to give a formula for the essential norm of the composition operator from $H^{2}$ to $H^{2}$ induced by $\varphi$. In the same paper Shapiro also introduced the generalized Nevanlinna counting functions $N_{\varphi, \gamma}$, defined for $\gamma>0$ by

$$
N_{\varphi, \gamma}(w)=N_{\gamma}(w)=\sum_{z \in \varphi^{-1}\{w\}}[\log (1 /|z|)]^{\gamma}, \quad w \in \mathbb{D} \backslash\{\varphi(0)\},
$$

in his work on composition operators from a weighted Bergman space to itself.

Subsequently, in $[\mathrm{Sm}]$, the first author used the growth of these counting functions to characterize when $C_{\varphi}: A_{\alpha}^{p} \rightarrow A_{\beta}^{q}$ is bounded or compact, when $q \geq p$. The Hardy spaces were included in these results by defining $A_{-1}^{p}$ to be $H^{p}$; see the comments following Lemma 2.1 below. A growth condition was also shown to be sufficient for boundedness and compactness in the remaining case, when $q<p$, in [Sm], but a characterization was left open. In this paper we show that an integrability condition on the counting functions provides that characterization.

1.1 Theorem. Let $0<q<p$ and $\alpha>-1$, and suppose $\varphi$ is an analytic self-map of $\mathbb{D}$. Then the following are equivalent.

(i) $C_{\varphi}: A_{\alpha}^{p} \rightarrow A_{\beta}^{q}$ is bounded;

(ii) $C_{\varphi}: A_{\alpha}^{p} \rightarrow A_{\beta}^{q}$ is compact;

(iii) $\frac{N_{\varphi, \beta+2}(z)}{\left(1-|z|^{2}\right)^{2+\alpha}} \in L^{\frac{p}{p-q}}\left(d A_{\alpha}\right)$.

We remark that the theorem fails for $\alpha=-1$. For example, if $\varphi(z)=z$, then $C_{\varphi}: H^{2} \rightarrow H^{1}$ is bounded but not compact, and condition 1.1(iii) fails. We also note that it is an easy consequence of Littlewood's Inequality, stated in $\S 2$ below, that condition 1.1(iii) holds for every analytic self-map $\varphi$ of $\mathbb{D}$ whenever $q / p<(1+\beta) /(1+\alpha)$. In particular, this holds if $\beta \geq \alpha$, since $q / p<1$. The following corollary is the case $\alpha=0$ and $\beta=-1$ of this theorem.

1.2 Corollary. Let $0<q<p$ and suppose $\varphi$ is an analytic self-map of $\mathbb{D}$. Then the following are equivalent.

(i) $C_{\varphi}: A^{p} \rightarrow H^{q}$ is bounded;

(ii) $C_{\varphi}: A^{p} \rightarrow H^{q}$ is compact;

(iii) $\frac{N_{\varphi, 1}(z)}{\left(1-|z|^{2}\right)^{2}} \in L^{\frac{p}{p-q}}(d A)$.

A natural question is suggested by this corollary: what operator theoretic property of $C_{\varphi}$ is characterized by the condition $N_{\varphi, 1}(z) /\left(1-|z|^{2}\right)^{2} \in L^{1}(d A)$ ? This has been recently answered by the first author and R. Zhao in Theorem 1.3 of [SZ], where it is shown that this condition is equivalent to $C_{\varphi}: \mathcal{B} \rightarrow H^{2}$ being bounded or compact. Here $\mathcal{B}$ denotes the Bloch space.

We present one application of Corollary 1.2 to polygonal maps. Let $P \subset \overline{\mathbb{D}}$ be a polygon and let $\pi / \eta$ be the largest angle at a vertex of $P$ that lies on $\partial \mathbb{D}$. It was proved as Theorem 6.7 of $[\mathrm{Sm}]$ that if $\varphi$ is an analytic self-map of $\mathbb{D}$ with $\varphi(D) \subset P$ and $\eta \geq 2 q / p \geq 2$, then $C_{\varphi}: A^{p} \rightarrow H^{q}$ is bounded. This conclusion is sharp in the 
sense that $C_{\varphi}$ need not be compact and the lower bound for $\eta$ cannot be increased. Using Corollary 1.2 we can now get a sharp result for the case $q<p$.

1.3 Theorem. Let $0<q<p$ and let $P$ and $\eta$ be as above. If $\varphi$ is an analytic self-map of $\mathbb{D}$ with $\varphi(\mathbb{D}) \subset p$ and $\eta>2 q / p$, then $C_{\varphi}: A^{p} \rightarrow H^{q}$ is bounded or equivalently compact. Moreover, this fails for $\eta \leq 2 q / p$.

We only sketch the proof; see the proof of Theorem 6.7 in $[\mathrm{Sm}]$ for more details. By Littlewood's Subordination Principle, it suffices to consider the case that $\varphi$ is a Riemann map from $\mathbb{D}$ onto $P$. Since $N_{\varphi, 1}$ is supported on $P$, we need only check that the integrability condition 1.1(iii) is satisfied at each of the finitely many vertices of $P$ on $\partial \mathbb{D}$. It is an easy consequence of the Schwarz Reflection Principle that $N_{\varphi, 1}(w)=O\left(\left(1-|w|^{2}\right)^{\eta}\right)$, as $|w| \rightarrow 1$, with these quantities comparable in an appropriate sector having vertex at the largest angle of $P$ on $\partial \mathbb{D}$. Thus, using polar coordinates at each vertex in turn, we see 1.1(iii) holds if and only if $(2-\eta) p /(p-q)<2$, or equivalently $\eta>2 q / p$.

Our proof of Theorem 1.1 uses the method D. H. Luecking used in [Lu] to establish a necessary condition for a measure $\mu$ to satisfy an inequality of the form

$$
\left(\int_{\mathbb{D}}\left|f^{(n)}\right|^{q} d \mu\right)^{1 / q} \leq C\|f\|_{A_{p}}
$$

where $q<p$. Luecking gives a characterization of the measures satisfying this inequality, but it is the necessity part of the argument that is the most interesting for us and that we employ here. In our work with composition operators mapping into $A_{\beta}^{q}$, we estimate integrals of the form

$$
\int_{\mathbb{D}}|f|^{q-2}\left|f^{\prime}\right|^{2} d \mu
$$

see Lemma 2.1 below. If we could assume $q=2$, then Luecking's result could be applied and the proof of Theorem 1.1 would be simpler. This reduction does not seem possible, but nevertheless Luecking's method applies. In this context we remark that it follows from the work of C. Horowitz that, for an integer $n \geq 1$ and $\alpha \geq 0, C_{\varphi}: A_{\alpha}^{p} \rightarrow A_{\beta}^{q}$ is bounded if and only if $C_{\varphi}: A_{\alpha}^{n p} \rightarrow A_{\beta}^{n q}$ is bounded. One direction is elementary, while the other uses Horowitz's factorization theorem for functions in $A_{\alpha}^{p}, \alpha \geq 0$; see Theorem 1 in $[\mathrm{H}]$. Even with this result it does not seem easy to make the reduction of assuming that $q=2$ when $\alpha \geq 0$.

In the next section we provide background material on the weighted Bergman spaces and the generalized counting functions. Khinchine's Inequality, which played a key role in $[\mathrm{Lu}]$ and will be used in our proof of Theorem 1.1, is also reviewed. Theorem 1.1 will then be proved in $\S 3$.

\section{BACKGROUND MATERIAL}

The utility of the generalized counting functions in studying composition operators between weighted Bergman spaces comes from the following lemma.

2.1 Lemma ([Sm, Proposition 2.4]). Let $\varphi$ be an analytic self-map of $D$ and let $f$ be analytic on $D$. Then, for $\gamma \geq-1$,

$$
\|f \circ \varphi\|_{A_{\gamma}^{p}}^{p} \approx|f(\varphi(0))|^{p}+\int_{\mathbb{D}}|f|^{p-2}\left|f^{\prime}\right|^{2} N_{\varphi, \gamma+2} d A .
$$


Here the symbol " $\approx$ " means that the left-hand side is bounded above and below by constant multiples of the right-hand side, where the constants are positive and independent of $f$. The case $\gamma=-1$ requires some comment. Recall that the spaces $A_{\gamma}^{p}$ were initially defined just for $\gamma>-1$. The reason for this is that if $\gamma \leq-1$, then $d A_{\gamma}(\mathbb{D})=\infty$, and it is easy to see that $A_{\gamma}^{p}$ contains just the zero function. The right side of the display above, however, is well behaved with $\gamma=-1$. Indeed, it is a special case of C. S. Stanton's formula for integral means of analytic functions [St] (see also [ESS] and $[\mathrm{Sm}]$ ) that

$$
\|f \circ \varphi\|_{H^{p}}^{p}=|f(\varphi(0))|^{p}+\frac{p^{2}}{2} \int_{\mathbb{D}}|f|^{p-2}\left|f^{\prime}\right|^{2} N_{\varphi, 1} d A .
$$

Comparing this formula to the statement of Lemma 2.1, we see that the definition $A_{-1}^{p}=H^{p}$ allows us to include the Hardy spaces in our results.

We now collect some properties of the generalized counting functions that we will require. The first is a bound for $N_{\psi, \gamma}$ known as Littlewood's Inequality. This was established by Littlewood for $\gamma=1$ in [Li], and was extended to $\gamma>1$ by Shapiro; see Proposition 6.3 in [Sh].

Littlewood's Inequality. Let $\psi$ be an analytic self-map of $\mathbb{D}$ and let $\gamma \geq 1$. If $\psi(0)=0$, then

$$
N_{\psi, \gamma}(z) \leq(\log (1 /|z|))^{\gamma}, \quad z \in \mathbb{D} \backslash\{0\} .
$$

From now on, for $\lambda \in \mathbb{D}$ let $\sigma_{\lambda}(w)=(\lambda-w) /(1-\bar{\lambda} w)$ be the automorphism of $\mathbb{D}$ that exchanges 0 and $\lambda$. The next lemma shows how the counting functions transform under composition with these automorphisms. It is an easy consequence of the definition of the counting functions; see Lemma 4.2 in [Sm].

2.2 Lemma. Let $\psi$ be an analytic self-map of $\mathbb{D}$ and let $\lambda \in D$. Then

$$
\left(N_{\psi, \gamma}\right) \circ \sigma_{\lambda}=N_{\sigma_{\lambda} \circ \psi, \gamma} .
$$

An important property of these counting functions is that they satisfy a subharmonic mean value property. This was proved for the classical case $\gamma=1$ in [ESS] and for the generalized counting functions by Shapiro as Corollary 6.7 of [Sh].

Subharmonic Mean Value Property. Let $\psi$ be an analytic self-map of $\mathbb{D}$ and let $\gamma \geq 1$. If $\psi(0) \neq 0$ and $0<r<|\psi(0)|$, then

$$
N_{\psi, \gamma}(0) \leq \frac{1}{r^{2}} \int_{r D} N_{\psi, \gamma} d A .
$$

The lemma below is a conformally invariant formulation of this property that will be used in proving Theorem 1.1. We use $D(\lambda, \delta)$ to denote the pseudohyperbolic disk with center $\lambda$ and radius $\delta$ :

$$
D(\lambda, \delta)=\left\{w:\left|\sigma_{\lambda}(w)\right|<\delta\right\} .
$$

2.3 Lemma. Let $\psi$ be an analytic self-map of $\mathbb{D}$ satisfying $\psi(0)=0$ and let $\gamma \geq 1$. There is a constant $C$ such that if $1>|w|>1 / 4$, then

$$
N_{\psi, \gamma}(w) \leq \frac{C}{\left(1-|w|^{2}\right)^{2}} \int_{D\left(w, \frac{1}{4}\right)} N_{\psi, \gamma} d A
$$


Proof. By applying Lemma 2.2, the Subharmonic Mean Value Property and then Lemma 2.2 again, we have

$$
N_{\psi, \gamma}(w)=N_{\sigma_{w} \circ \psi, \gamma}(0) \leq 16 \int_{\frac{1}{4} \mathbb{D}} N_{\sigma_{w} \circ \psi, \gamma} d A=16 \int_{\frac{1}{4} \mathbb{D}} N_{\psi, \gamma} \circ \sigma_{w} d A .
$$

Note that the application of the Subharmonic Mean Value Property used the assumption that $\left|\sigma_{w} \circ \psi(0)\right|=|w|>1 / 4$. The change of variable $\zeta=\sigma_{w}(z)$, along with the estimate $\left|\sigma_{w}^{\prime}(\zeta)\right| \approx\left(1-|w|^{2}\right)^{-1}$ for $\zeta \in D(w, 1 / 4)$, now shows

$$
N_{\psi, \gamma}(w) \leq \frac{C}{\left(1-|w|^{2}\right)^{2}} \int_{D\left(w, \frac{1}{4}\right)} N_{\psi, \gamma} d A .
$$

2.4 Lemma. There is a constant $C$ such that, for every function $f$ analytic on $\mathbb{D}$ and all $\lambda \in \mathbb{D}$,

$$
\int_{D(\lambda, 1 / 4)}|f|^{q-2}\left|f^{\prime}\right|^{2} d A \leq \frac{C}{\left(1-|\lambda|^{2}\right)^{2}} \int_{D(\lambda, 1 / 2)}|f|^{q} d A
$$

Proof. By making the change of variable $w=\sigma_{\lambda}(z)$ and using that $\left|\sigma_{\lambda}^{\prime}(z)\right| \approx$ $\left(1-|\lambda|^{2}\right)^{-1}$ for $z \in D(\lambda, 1 / 2)$, we may assume that $\lambda=0$. After this reduction, the given estimate is an easy consequence of Lemma 2.1, applied with $\gamma=0$ and $\varphi(z)=z / 2$.

Our proof of Theorem 1.1 uses Khinchine's Inequality. This inequality involves the Rademacher functions $\left\{r_{n}(t)\right\}$, defined as follows:

$$
\begin{aligned}
& r_{0}(t)= \begin{cases}1, & 0 \leq t-[t]<\frac{1}{2}, \\
-1, & \frac{1}{2} \leq t-[t]<1 ;\end{cases} \\
& r_{n}(t)=r_{0}\left(2^{n} t\right), \quad n>0 .
\end{aligned}
$$

Khinchine's Inequality. Let $0<p<\infty$ and let $\left\{c_{j}\right\}_{j=1}^{m}$ be complex numbers. There are constants $0<b_{p} \leq B_{p}<\infty$, depending only on $p$, such that

$$
b_{p}\left(\sum_{j=1}^{m}\left|c_{j}\right|^{2}\right)^{p / 2} \leq \int_{0}^{1}\left|\sum_{j=1}^{m} c_{j} r_{j}(t)\right|^{p} d t \leq B_{p}\left(\sum_{j=1}^{m}\left|c_{j}\right|^{2}\right)^{p / 2} .
$$

A final ingredient of our proof of Theorem 1.1 is that the Hardy-Littlewood maximal function can be used to give a bound for the average of a function over a pseudohyperbolic disk. Let $M[f]$ denote the Hardy-Littlewood maximal function for $f$, i.e.

$$
M[f](z)=\sup _{\delta>0} \frac{1}{A(B(z, \delta))} \int_{B(z, \delta)}|f| d A .
$$

Here $B(z, \delta)=\{w:|z-w|<\delta\}$ and $f$ is extended to be 0 outside its original domain of definition. Since there is a constant $C$ such that $D(\lambda, 1 / 2) \subset B\left(\lambda, C\left(1-|\lambda|^{2}\right)\right)$ for all $\lambda \in \mathbb{D}$, it follows that

$$
\frac{1}{\left(1-|\lambda|^{2}\right)^{2}} \int_{D(\lambda, 1 / 2)}|f| d A \leq C \cdot M[f](\lambda) .
$$




\section{Proof of the MAIN Result}

Proof of Theorem 1.1. We begin by observing that it is enough to prove the theorem under the assumption that $\varphi(0)=0$. To see this, replace $\varphi$ with $\sigma_{\varphi(0)} \circ \varphi$, noting that $C_{\varphi}$ is bounded or compact if and only if $C_{\varphi} C_{\sigma_{\varphi}(0)}$ is bounded or compact, since $C_{\sigma_{\varphi(0)}}$ is bounded and invertible on every weighted Bergman space. Also, $N_{\sigma_{\varphi(0)} \circ \varphi, \beta+2}=N_{\varphi, \beta+2} \circ \sigma_{\varphi(0)}$, and it is easy to verify that this function satisfies condition 1.1(iii) if and only if $N_{\varphi, \beta+2}$ does.

We first assume that $\frac{N_{\varphi, \beta+2}}{\left(1-|z|^{2}\right)^{2+\alpha}} \in L^{\frac{p}{p-q}}\left(d A_{\alpha}\right)$ and show $C_{\varphi}: A_{\alpha}^{p} \rightarrow A_{\beta}^{q}$ is bounded. From the Closed Graph Theorem, it suffices to show that $C_{\varphi}$ maps $A_{\alpha}^{p}$ into $A_{\beta}^{q}$. By Lemma 2.1, this means we must show that if $f \in A_{\alpha}^{p}$, then

$$
\int_{\mathbb{D}}|f|^{q-2}\left|f^{\prime}\right|^{2} N_{\varphi, \beta+2} d A<\infty .
$$

To see this, we work with the integrals over $\frac{1}{4} \mathbb{D}$ and $\mathbb{D} \backslash \frac{1}{4} \mathbb{D}$ separately. For the integral over $\frac{1}{4} \mathbb{D}$, we recall that Littlewood's Inequality asserts

$$
N_{\varphi, \beta+2}(z) \leq(\log (1 /|z|))^{\beta+2} .
$$

Also, for the function $\psi(z)=z / 2$ we have

$$
N_{\psi, \beta+2}(z)=(\log (1 / 2|z|))^{\beta+2}, \quad|z| \leq 1 / 2,
$$

and so

$$
N_{\varphi, \beta+2}(z) \leq 2^{\beta+2} N_{\psi, \beta+2}(z), \quad|z| \leq 1 / 4 .
$$

This estimate and Lemma 2.1 therefore give

$$
\int_{\frac{1}{4} \mathbb{D}}|f|^{q-2}\left|f^{\prime}\right|^{2} N_{\varphi, \beta+2} d A \leq 2^{\beta+2} \int_{\frac{1}{4} \mathbb{D}}|f|^{q-2}\left|f^{\prime}\right|^{2} N_{\psi, \beta+2} d A \leq C \int_{\mathbb{D}}|f \circ \psi|^{q} d A_{\beta} .
$$

Since the $f$ is bounded on $\psi(\mathbb{D})$, it follows that

$$
\int_{\frac{1}{4} \mathbb{D}}|f|^{q-2}\left|f^{\prime}\right|^{2} N_{\varphi, \beta+2} d A<\infty .
$$

Next, using Lemma 2.3 for $w \in \mathbb{D} \backslash \frac{1}{4} \mathbb{D}$,

$$
\begin{aligned}
\int_{\mathbb{D} \backslash \frac{1}{4} \mathbb{D}}|f|^{q-2}\left|f^{\prime}\right|^{2} N_{\varphi, \beta+2} d A & \\
& \leq C \int_{\mathbb{D}}|f(w)|^{q-2}\left|f^{\prime}(w)\right|^{2} \frac{1}{\left(1-|w|^{2}\right)^{2}} \int_{D\left(w, \frac{1}{4}\right)} N_{\varphi, \beta+2}(z) d A(z) d A(w) .
\end{aligned}
$$

We now apply Fubini's Theorem, using that $\chi_{D(w, 1 / 4)}(z)=\chi_{D(z, 1 / 4)}(w)$ and 1 $|w|^{2} \approx 1-|z|^{2}$ for $w \in D(z, 1 / 4)$, and then Lemma 2.4 to get that the above is less than

$$
\begin{array}{rl}
C \int_{\mathbb{D}} \int_{D\left(z, \frac{1}{4}\right)}|f(w)|^{q-2}\left|f^{\prime}(w)\right|^{2} & d A(w) \frac{N_{\varphi, \beta+2}(z)}{\left(1-|z|^{2} \mid\right)^{2}} d A(z) \\
\leq C \int_{\mathbb{D}} \int_{D\left(z, \frac{1}{2}\right)}|f(w)|^{q} d A(w) \frac{N_{\varphi, \beta+2}(z)}{\left(1-|z|^{2}\right)^{4}} d A(z)
\end{array}
$$


Another application of Fubini's Theorem thus gives

$$
\int_{\mathbb{D} \backslash \frac{1}{4} \mathbb{D}}|f|^{q-2}\left|f^{\prime}\right|^{2} N_{\varphi, \beta+2} d A \leq C \int_{\mathbb{D}}|f|^{q} H d A_{\alpha},
$$

where

$$
H(w)=\int_{D\left(w, \frac{1}{2}\right)} \frac{N_{\varphi, \beta+2}(z)}{\left(1-|z|^{2}\right)^{4}} d A(z) \cdot \frac{1}{\left(1-|w|^{2}\right)^{\alpha}} .
$$

The lower bound of $M[f]$ noted in (2.1) yields that

$$
H(w) \leq C \cdot M\left[\frac{N_{\varphi, \beta+2}}{\left(1-|z|^{2}\right)^{\frac{q}{p} \alpha+2}}\right](w) \cdot \frac{1}{\left(1-|w|^{2}\right)^{\frac{p-q}{p} \alpha}} .
$$

The well known estimate that, for $s>1,\|M[f]\|_{s} \leq C\|f\|_{s}$, and the assumption that condition 1.1(iii) holds, now show

$$
\begin{aligned}
\int H(w)^{\frac{p}{p-q}} d A_{\alpha}(w) & \leq C\left\|M\left[\left(1-|z|^{2}\right)^{-\frac{q}{p} \alpha-2} N_{\varphi, \beta+2}(z)\right]\right\|_{\frac{p}{p-q}}^{\frac{p}{p-q}} \\
& \leq C\left\|\frac{N_{\varphi, \beta+2}}{\left(1-|z|^{2}\right)^{2+\alpha}}\right\|_{L^{\frac{p}{p-q}}}^{\frac{p}{p-q}}\left(d A_{a}\right) \\
& <\infty
\end{aligned}
$$

and so $H \in L^{\frac{p}{p-q}}\left(d A_{\alpha}\right)$. Since we are assuming that $\alpha>-1$ and $f \in A_{\alpha}^{p}$, $\int|f|^{p} d A_{\alpha}<\infty$. Thus it is now a consequence of Hölder's inequality and (3.4) that

$$
\int_{\mathbb{D} \backslash \frac{1}{4} \mathbb{D}}|f|^{q-2}\left|f^{\prime}\right|^{2} N_{\varphi, \beta+2} d A<\infty .
$$

Combined with (3.3) this shows that (3.1) holds, and therefore that $C_{\varphi}: A_{\alpha}^{p} \rightarrow A_{\beta}^{q}$ is bounded. This completes the proof that condition 1.1(iii) implies condition 1.1(i).

We now show that $\frac{N_{\varphi, \beta+2}}{\left(1-|z|^{2}\right)^{2+\alpha}} \in L^{\frac{p}{p-q}}\left(d A_{\alpha}\right)$ actually implies that $C_{\varphi}: A_{\alpha}^{p} \rightarrow$ $A_{\beta}^{q}$ is compact. We must prove that if $\left\{f_{n}\right\} \subset A_{\alpha}^{p}$ and $\left\|f_{n}\right\|_{A_{\alpha}^{p}} \leq 1$, then there is a subsequence of $\left\{C_{\varphi} f_{n}\right\}$ that converges in $A_{\beta}^{q}$. Since functions in the unit ball of $A_{\alpha}^{p}$ are uniformly bounded on compact subsets of $\mathbb{D}$ (see Lemma 2.5 in [Sm]), they comprise a normal family. Hence by passing to a subsequence we may assume that $\left\{f_{n}\right\}$ converges to $f$ uniformly on compact subsets of $\mathbb{D}$, and by considering integrals over $r \mathbb{D}$ and letting $r$ increase to 1 we see that $f \in A_{\alpha}^{p}$. Since we have shown that $C_{\varphi}: A_{\alpha}^{p} \rightarrow A_{\beta}^{q}$ is bounded, $C_{\varphi} f \in A_{\beta}^{q}$. Thus by subtracting $f$ from each element of the sequence $\left\{f_{n}\right\}$, we may assume that $\left\{f_{n}\right\}$ converges to 0 uniformly on compact subsets of $\mathbb{D}$, and it suffices to show that $\lim _{n \rightarrow \infty}\left\|C_{\varphi} f_{n}\right\|_{A_{\beta}^{q}}=0$. By Lemma 2.1, this means that we must show

$$
\lim _{n \rightarrow \infty} \int_{\mathbb{D}}\left|f_{n}\right|^{q-2}\left|f_{n}^{\prime}\right|^{2} N_{\varphi, \beta+2} d A=0 .
$$

The proof now follows the argument used to show $C_{\varphi}$ was bounded, by considering the integrals over $\frac{1}{4} \mathbb{D}$ and $\mathbb{D} \backslash \frac{1}{4} \mathbb{D}$ separately. By applying (3.2) with $f$ replaced by $f_{n}$, we get that

$$
\lim _{n \rightarrow \infty} \int_{\frac{1}{4} \mathbb{D}}\left|f_{n}\right|^{q-2}\left|f_{n}^{\prime}\right|^{2} N_{\varphi, \beta+2} d A=0
$$


since the sequence $\left\{f_{n}\right\}$ converges to 0 uniformly on $\psi(\mathbb{D})$. For the integral over $\mathbb{D} \backslash \frac{1}{4} \mathbb{D}$, it is a simple exercise using Hölder's inequality applied to (3.4) to show that since $H \in L^{\frac{p}{p-q}}\left(d A_{\alpha}\right)$, the assumptions about the sequence $\left\{f_{n}\right\}$ imply

$$
\lim _{n \rightarrow \infty} \int_{\mathbb{D} \backslash \frac{1}{4} \mathbb{D}}\left|f_{n}\right|^{q-2}\left|f_{n}^{\prime}\right|^{2} N_{\varphi, \beta+2} d A=0 .
$$

Combined with (3.6) this shows that (3.5) holds, and therefore that $C_{\varphi}: A_{\alpha}^{p} \rightarrow A_{\beta}^{q}$ is compact. This completes the proof that condition 1.1(iii) implies condition 1.1(ii).

Since a compact operator is bounded, it remains to prove that condition 1.1(i) implies condition 1.1(iii). This proof involves ideas from the theory of atomic decomposition of the $A_{\alpha}^{p}$ spaces. A sequence of points $\left\{z_{n}\right\} \subset \mathbb{D}$ is said to have a positive separation constant if the pseudohyperbolic distance between points of the sequence is bounded below, i.e.

$$
\inf _{n \neq m}\left|\sigma_{z_{n}}\left(z_{m}\right)\right|>0
$$

Let $f(z)=\sum_{n} a_{n} k_{n}(z)$, where

$$
k_{n}(z)=\bar{z}_{n}^{-1} \frac{\left(1-\left|z_{n}\right|^{2}\right)^{M-2 / p-\alpha / p}}{\left(1-\bar{z}_{n} z\right)^{M}}
$$

and $M>(2+\alpha) \max (1,1 / p)$. It is known that if $\left\{z_{n}\right\}$ has a positive separation constant, then there is a constant $C$ such that

$$
\|f\|_{A_{\alpha}^{p}} \leq C\left(\sum_{n}\left|a_{n}\right|^{p}\right)^{1 / p}
$$

see Theorem 2.2 in [Ro]. (We note the assumption that $\alpha>-1$ was used here.) We apply this inequality with $a_{n}$ replaced by $a_{n} r_{n}(t)$, where the $r_{n}$ are the Rademacher functions introduced in $\S 2$. Suppose that $C_{\varphi}: A_{\alpha}^{p} \rightarrow A_{\beta}^{q}$ is bounded, and for now assume that $q \geq 2$. Then, recalling that $\left|r_{n}(t)\right|=1$,

$$
\int_{\mathbb{D}}\left|\sum_{n} a_{n} r_{n}(t) k_{n} \circ \varphi\right|^{q} d A_{\beta} \leq\left\|C_{\varphi}\right\|^{q}\|f\|_{A_{\alpha}^{p}}^{q} \leq C\left(\sum_{n}\left|a_{n}\right|^{p}\right)^{q / p} .
$$

Integrating this inequality from 0 to 1 with respect to $t$, we get

$$
\int_{\mathbb{D}} \sum_{n}\left|a_{n}\right|^{q}\left|k_{n} \circ \varphi\right|^{q} d A_{\beta} \leq \int_{\mathbb{D}}\left(\sum_{n}\left|a_{n}\right|^{2}\left|k_{n} \circ \varphi\right|^{2}\right)^{q / 2} d A_{\beta} \leq C\left(\sum_{n}\left|a_{n}\right|^{p}\right)^{q / p} .
$$

The first inequality just used the assumption that $q \geq 2$, and the second inequality followed from Khinchine's Inequality. From Lemma 2.1, we conclude that

$$
\sum_{n}\left|a_{n}\right|^{q} \int_{\mathbb{D}}\left|k_{n}\right|^{q-2}\left|k_{n}^{\prime}\right|^{2} N_{\varphi, \beta+2} d A \leq C\left(\sum_{n}\left|a_{n}\right|^{p}\right)^{q / p} .
$$

It is easily verified that

$$
\frac{1}{\left(1-\left|z_{n}\right|^{2}\right)^{(2+\alpha) \frac{q}{p}+2}} \leq C\left|k_{n}\right|^{q-2}\left|k_{n}^{\prime}\right|^{2}
$$


on $D\left(z_{n}, 1 / 2\right)$, and thus

$$
\sum_{n}\left|a_{n}\right|^{q} \frac{\int_{D\left(z_{n}, \frac{1}{2}\right)} N_{\varphi, \beta+2} d A}{\left(1-\left|z_{n}\right|^{2}\right)^{(2+\alpha) \frac{q}{p}+2}} \leq C\left(\sum_{n}\left|a_{n}\right|^{p}\right)^{q / p} .
$$

The argument now proceeds exactly as in $[\mathrm{Lu}]$. We include a sketch of the argument for completeness; see [Lu] for further details. From the inequality above, we conclude that the sequence

$$
\frac{\int_{D\left(z_{n}, \frac{1}{2}\right)} N_{\varphi, \beta+2} d A}{\left(1-\left|z_{n}\right|^{2}\right)^{(2+\alpha) \frac{q}{p}+2}}
$$

belongs to the dual of $\ell^{p / q}$, or equivalently

$$
\sum_{n}\left(\frac{\int_{D\left(z_{n}, \frac{1}{2}\right)} N_{\varphi, \beta+2} d A}{\left(1-\left|z_{n}\right|^{2}\right)^{4+\alpha \frac{q}{p}}}\right)^{\frac{p}{p-q}} A\left(D\left(z_{n}, \frac{1}{2}\right)\right)<\infty .
$$

To get the integrability condition we require from this, we choose the sequence $\left\{z_{n}\right\}$ so that it has positive separation constant and so that the disks $D\left(z_{n}, 1 / 4\right)$ cover D. Then

$$
\begin{aligned}
\int_{\mathbb{D}}\left(\frac{\int_{D\left(z, \frac{1}{4}\right)} N_{\varphi, \beta+2} d A}{\left(1-|z|^{2}\right)^{4+\frac{q}{p} \alpha}}\right)^{\frac{p}{p-q}} d A & \leq \sum \int_{D\left(z_{n}, 1 / 4\right)}\left(\frac{\int_{D\left(z, \frac{1}{4}\right)} N_{\varphi, \beta+2} d A}{\left(1-|z|^{2}\right)^{4+\frac{q}{p} \alpha}}\right)^{\frac{p}{p-q}} d A \\
& \leq C \sum_{n}\left(\frac{\int_{D\left(z_{n}, \frac{1}{2}\right)} N_{\varphi, \beta+2} d A}{\left(1-\left|z_{n}\right|^{2}\right)^{4+\alpha \frac{q}{p}}}\right)^{\frac{p}{p-q}} A\left(D\left(z_{n}, \frac{1}{4}\right)\right),
\end{aligned}
$$

which is finite by the preceding displayed inequality, and so

$$
\frac{\int_{D\left(z, \frac{1}{4}\right)} N_{\varphi, \beta+2} d A}{\left(1-|z|^{2}\right)^{4+\frac{q}{p} \alpha}} \in L^{\frac{p}{p-q}}(d A) .
$$

Since the integrability on $\frac{1}{4} \mathbb{D}$ is clear, Lemma 2.3 now shows that

$$
\frac{N_{\varphi, \beta+2}}{\left(1-|z|^{2}\right)^{2+\frac{q}{p} \alpha}} \in L^{\frac{p}{p-q}}(d A)
$$

and it is easily checked that this is equivalent to

$$
\frac{N_{\varphi, \beta+2}}{\left(1-|z|^{2}\right)^{2+\alpha}} \in L^{\frac{p}{p-q}}\left(d A_{\alpha}\right) \text {. }
$$

This completes the proof that condition 1.1(i) implies condition 1.1(iii) in the case that $q \geq 2$. If $q<2$, choose an integer $m$ satisfying $m q \geq 2$. Assume that $1.1(\mathrm{i})$ holds, i.e. $C_{\varphi}: A_{\alpha}^{p} \rightarrow A_{\beta}^{q}$ is bounded, and let $f \in A_{\alpha}^{m p}$. Then $f^{m} \in A_{\alpha}^{p}$, and so $C_{\varphi} f^{m}=(f \circ \varphi)^{m} \in A_{\beta}^{q}$. This is equivalent to $f \circ \varphi \in A_{\beta}^{m q}$, and so $C_{\varphi}: A_{\alpha}^{m p} \rightarrow A_{\beta}^{m q}$ is bounded by the Closed Graph Theorem. Since $m q \geq 2$, we get that

$$
\frac{N_{\varphi, \beta+2}}{\left(1-|z|^{2}\right)^{2+\alpha}} \in L^{\frac{m p}{m p-m q}}\left(d A_{\alpha}\right)=L^{\frac{p}{p-q}}\left(d A_{\alpha}\right),
$$

as required, and the proof of the theorem is complete. 


\section{REFERENCES}

[ESS] Essén, M., Shea, D.F. and Stanton, C.S., A value-distribution criterion for the class $L \log L$, and some related questions, Ann. Inst. Fourier (Grenoble) 35 (1985), 127-150. MR 87e:30041

[H] Horowitz, C., Factorization theorems for functions in the Bergman spaces, Duke Math. J. 44 (1977), 201-213. MR 55:681

[HJ] Hunziker, H. and Jarchow, H., Composition operators which improve integrability, Math. Nachr. 152 (1991), 83-91. MR 93d:47061

[Li] Littlewood, J.E., On inequalities in the theory of functions, Proc. London Math. Soc. 23 (1925), 481-519.

[Lu] Luecking, D. H., Embedding theorems for spaces of analytic functions via Khinchine's inequality, Michigan Math. J. 40 (1993), 333-358. MR 94e:46046

[MS] MacCluer, B.D. and Shapiro, J.H., Angular derivatives and compact composition operators on the Hardy and Bergman spaces, Canad. J. Math. 38 (1986), 878-906. MR 87h:47048

[Ri] Riedl, R., Composition operators and geometric properties of analytic functions, Thesis, Universität Zurich (1994).

[Ro] Rockberg, R., Decomposition theorems for Bergman spaces and their applications, in Operators and Function Theory (S.C. Power, editor), D. Reidel, Dordrecht, 1985, pp. 225-277.

[Sh] Shapiro, J.H., The essential norm of a composition operator, Annals of Math. 127 (1987), 375-404. MR 88c:47058

[Sm] Smith, W., Composition operators between Bergman and Hardy spaces, Trans. Amer. Math. Soc. 348 (1996), 2331-2348. MR 96i:47056

[SZ] Smith, W. and Zhao, R., Composition operators mapping into the $Q_{p}$ spaces, preprint.

[St] Stanton, C. S., Counting functions and majorization for Jensen measures, Pacific J. Math. 125 (1986), 459-468. MR 88c:32002

Department of Mathematics, University of Hawail, Honolulu, Hawail 96822

E-mail address: wayne@math.hawaii.edu

E-mail address: yang@math.hawaii.edu 